\title{
Medición de la calidad del servicio de proveedores de TIC: proposición de una escala
}

José Lora Andosilla*

Beatriz Helena Díaz Pinzón **

Fecha de recepción: 13 de septiembre de 2013

Fecha de aprobación: 20 de octubre de 2013

Pp.63 a 100.

\section{Resumen}

El presente artículo, se centra en la construcción de una escala para medir la calidad del servicio en proveedores de tecnologías de la información y las comunicaciones (TIC). Como resultado de la aplicación de una metodología de ocho pasos, se obtuvo una escala de tres dimensiones y 20 ítems. Las dimensiones resultantes son: interés en el cliente, experticia de los empleados y cumplimiento del proveedor.

La aplicación de la escala, permite diagnosticar la calidad del servicio percibida por los clientes y con base en los resultados, diseñar y ejecutar estrategias que permitan agregar valor a la organización y mejorar su competitividad en el mercado.

\section{Palabras clave}

Calidad del servicio, tecnología, escala, SERVPERF, SERVQUAL.

* Master en Administración, Universidad NAcional de Colombia.

** PhD. en Ciencias de Gestión, Universidad de Grenoble, Francia. 


\section{Measurement of the quality of ICT providers' services: a proposal of one useful scale}

\section{Abstract}

This article focuses on the construction of a scale which measures the quality of providers' services of Information and Communication Technologies (ICT). As a result of the application of an 8-step method, a three-dimensional scale and 20 items was obtained. The resulting dimensions are interest on the client, employees' expertise and providers' compliance. The application of this scale allows us to diagnose the quality of services as perceived by customers receiving an ICT provider's services, and focusing on outcomes to design and implement strategies to provide added value to organizations and to improve its competitiveness in the market.

\section{KEY WORDS}

Quality of Services, Technology, Scale, SERVPERF and SERCQUAL. 


\section{Qualité du service des fournisseurs de TIC: proposition d'une échelle de mesure}

\section{RÉSUMÉ}

Cet article se centre sur la construction d'une échelle mesurant la qualité du service des fournisseurs de technologies de l'information et de la communication (TIC) après la mise en place d'une méthodologie en 8 étapes. Une échelle de trois paramètres et 20 éléments a pu être adoptée. Les paramètres obtenus sont : l'expertise de l'employé, la conformité du fournisseur et l'intérêt du client. La mise en place de l'échelle de mesure permet de diagnostiquer la qualité du service perçu par les clients d'un fournisseur de TIC et sur la base de ces résultats, mettre en place des stratégies offrant une valeur ajoutée à l'organisation et améliorant sa compétitivité.

\section{Mots-clés}

Qualité de Service, Technologie, Echelle, SERVQUAL, SERVPERF 


\section{Medição da qualidade do serviço de fornecedores de TIC: proposta de uma escala}

\section{ReSUMO}

Este artigo centra-se na construção de uma escala para medir a qualidade do serviço em fornecedores de serviços de tecnologias da informação e comunicações (TIC). Como resultado da aplicação de uma metodologia de 8 passos obteve-se uma escala de três dimensões e 20 itens. As dimensões resultantes são: interesse no cliente, experiência dos empregados e cumprimento do fornecedor. A aplicação da escala permite diagnosticara qualidade do serviço percebida pelos clientes de um fornecedor de serviços de TIC e baseados nos resultados, desenhar e executar estrategias que permitam acrescentar valor na organização e melhorar sua competitividade no mercado.

\section{PalaVRas-ChaVe}

Qualidade do serviço, Tecnologia, Escala, SERVPERF, SERVQUAL 


\section{Introducción}

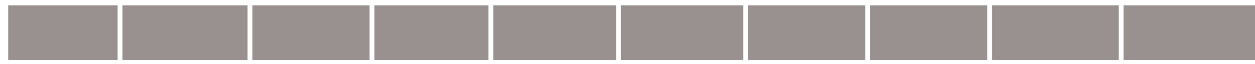

L

a contratación de proveedores de servicios de Tecnologías de la Información y las Telecomunicaciones (TIC), se ha hecho muy común desde mediados de 1980 y ha continuado su crecimiento hasta años recientes (Beasley, Bradford y Dehning, 2009).

Se define el servicio de TIC, como toda aquella actividad que usa tecnologías de la información y las comunicaciones para capturar, transportar, procesar, almacenar y presentar información digitalmente y para beneficiar a sus usuarios (Riascos, 2008) (Laudon y Laudon, 2008), (Paños, 1999), (Duque, 2005). Dentro de los servicios de TIC más significativos se pueden mencionar: la administración de centros de datos, las operaciones, el mantenimiento de Hardware, el mantenimiento de Software, las comunicaciones y el desarrollo de aplicaciones (Kishore, Rao, Nam, Rajagopalan y Chaudhury, 2003)

En las organizaciones, estos servicios de TIC son suministrados por proveedores internos y externos. Los internos, se refieren al empleado o grupo de personas que sirven a la organización y que pertenecen a un área o departamento dentro de esta. Los proveedores externos, son empresas o personas ajenas a la organización que suministran servicios de TIC mediante un acuerdo oral o escrito.

De acuerdo a Grover, Joong Cheon, y T.C. Teng (1996) las empresas contratan proveedores externos para alcanzar beneficios estratégicos, económicos y tecnológicos, los cuales, les permiten enfocarse en su negocio principal y mejorar su competitividad en el mercado. Adicionalmente, estos servicios les permiten aprovechar la experiencia del proveedor, disminuir costos y evitar el riesgo de obsolescencia tecnológica (Grover, Joong Cheon, y T.C. Teng, 1996). 
Centrarse en la calidad del servicio, le permite a un proveedor externo, aumentar su capacidad competitiva y crecimiento económico (Camisón, 1996; Devlin y Dong, 1994; Sureshghandar, Chandrasekharan y Anantharaman, 2002; Colmenares y Saavedra, 2007); satisfacer al cliente (Cronin y Taylor, 1992), generar intenciones de compra, disponibilidad del cliente a pagar más por el servicio y fidelidad a la empresa (Parasuraman, Zeithaml, y Berry, 1996). Experiencias insatisfactorias con proveedores de TIC, pueden ocasionar rompimiento de relaciones comerciales y en algunos casos demandas legales (Lora, 2009).

La presente investigación propone construir una escala que permita medir la calidad percibida del servicio de proveedores de TIC. 


\section{La calidad del servicio percibida: sus principales modelos y su aplicación en sistemas de información}

a calidad del servicio percibida, es una actitud relacionada con la superioridad de un servicio, la cual es subjetiva y multidimensional (Duque, 2005, 2006; Colmenares y Saavedra, 2007; Civera, 2008).

Diversos modelos de la calidad del servicio se han propuesto, pero de acuerdo a Brogowicz et al. (1990) y Duque (2005), el modelo nórdico de Gronroos (1978, 1982) y el americano de Parasuraman, Zeithaml y Berry (1985, 1988), han sido los más utilizados. Estos, han servido de base para el desarrollo de una gran porción de la literatura académica en el área y sus respectivos instrumentos de medición, han demostrado suficiente confiabilidad y validez en diferentes contextos (Duque, 2005).

En la gestión de sistemas de información, la literatura presenta principalmente, dos modelos. Por un lado, se tiene el IS SERVQUAL, de Pitt, Watson y Kavan (1995), el cual es una adaptación del SERVQUAL de Parasuraman, Zeithaml y Berry $(1985,1988)$; se propuso para adicionarle una nueva dimensión al modelo de éxito de los sistemas de información de DeLone y McLean (1992), el cual no se orientaba al servicio. De otra parte, está el modelo IS SERVPERF de Van Dyke, Kappelman y Prybutok (1997), basado en el SERVPERF de Cronin y Taylor (1992, 1994) y que propone como una mejor alternativa al IS SERVQUAL de Pitt, Watson, y Kavan (1995).

Ambos modelos están compuestos por cinco dimensiones:

- Elementos tangibles: se refiere a la apariencia física de las instalaciones, equipos, personal y al grado de actualización de Hardware y Software. 
- Fiabilidad o confiabilidad: habilidad para ejecutar el servicio prometido de manera confiable y cuidadosa desde la primera vez.

- Capacidad de respuesta o responsabilidad: disposición de ayudar y servir rápidamente a los clientes.

- Seguridad: habilidades y conocimientos de los empleados para inspirar credibilidad en el servicio que prestan.

- Empatía: atención individualizada que ofrecen las empresas a sus clientes.

Así mismo, cada uno de los modelos tiene su propio instrumento de medición, que han demostrado buen grado de validez y confianza.

A pesar de las similitudes, también se presentan grandes diferencias entre el IS SERVQUAL e IS SERVPERF, las cuales han servido de base para las críticas que han hecho defensores de un modelo sobre el otro. De acuerdo con lo anterior, a continuación se presenta una comparación entre ellos.

\subsection{Comparativo entre los modelos IS SERVQUAL e IS SERVPERF}

Con base en Van Dyke, Kappelman y Prybutok (1997), encontramos dos tipos de críticas hechas a los modelos: unas conceptuales y otras empíricas.

\subsubsection{Elementos conceptuales}

Diversos autores afirman que la calidad del servicio es una actitud (Parasuraman, Zeithaml y Berry 1985, 1988; Cronin y Taylor, 1992, 1994). Es así como, sugieren que debe basarse en modelos actitudinales (Cronin y Taylor, 1992), tal como lo hace el IS SERVPERF a diferencia del IS SERVQUAL, que toma como base el paradigma de la desconfirmación ${ }^{1}$ de Oliver (1980).

\footnotetext{
${ }^{1}$ Se refiere a la diferencia entre las expectativas que tiene un consumidor sobre un producto o servicio y sus percepciones después de haberlo consumirlo.
} 
Para Van Dyke, Kappelman y Prybutok (1997), es simplista medir todo el proceso cognitivo por el cual se evalúa la calidad del servicio percibido, basándose solo en el paradigma de la desconfirmación, por lo tanto sugieren que es mejor hacerlo a partir de las percepciones de las personas, debido a que el juicio que estas emitan sobre la calidad del servicio que perciban, provendrá directamente del proceso cognitivo que realicen para evaluarla. Sobre esta crítica, Pitt, Watson y Kavan (1997), manifestaron que la utilización del paradigma de la desconfirmación en el modelo IS SERVQUAL, surge de la investigación exploratoria hecha por Parasuraman, Zeithaml y Berry (1985), quienes adoptaron ese enfoque debido a las brechas o vacios que encontraron entre clientes y proveedores, las cuales dieron origen a su modelo SERVQUAL.

Diversos autores afirman que el uso del paradigma de la desconfirmación, generaambigüedad al medir las expectativas. pues las personas las interpretan de distintas maneras (Teas, 1993, 1994, 1997; Van Dyke, Kappelman, y Prybutok, 1997 y Cronin y Taylor, 1992, 1994). De acuerdo a Van Dyke, Kappelman, y Prybutok (1997) con el modelo IS SERVPERF, solo se miden las percepciones de las personas sobre el servicio, lo cual elimina cualquier ambigüedad que se presente con la medición de las expectativas.

\subsubsection{Elementos empíricos}

Algunos trabajos señalan que la escala IS SERVPERF, presenta mayor confiabilidad y validez que la IS SERVQUAL (Kettinger y Lee, 1994; Van dyke et al., 1997; Babakus y Boller, 1992; Boulding et al., 1993; Cronin y Taylor, 1992; Parasuraman, Zeithaml, y Berry, 1994; Van Dyke, Kappelman, y Prybutok, 1997). Dado que el modelo IS SERVPERF mide la calidad del servicio a partir de las percepciones de las personas, el número de ítems de su escala de medición se reduce a la mitad en comparación con la del IS SERVQUAL. De igual manera, los cálculos para obtener los resultados de la evaluación de la calidad del servicio percibido, se reducen porque no es necesario estimar la diferencia entre las medidas de las expectativas y las percepciones, tal como se hace con la escala IS SERVQUAL. 


\section{Metodología}

ara construir el instrumento de medición de la calidad del servicio de proveedores de servicios de TIC, se utilizó una metodología de ocho pasos basada en el paradigma de Churchill (1979), las recomendaciones de Zapata y Canet (2008), Uriel y Aldás (2005), Arribas (2004), Deng y Dart (1994), Hair, Anderson, Tatham y Black (1998), Steenkamp y Van Trijp (1991) y Anderson y Gerbing (1988) (ver figura 1).

Primero, en el paso 1 y 2 de la metodología, se seleccionó el modelo IS SERVPERF porque al no evaluar las expectativas del cliente sobre la calidad del servicio (como si lo hace el IS SERVQUAL), se evitan ambigüedades en la interpretación de sus ítems. Adicionalmente, este modelo mide directamente las percepciones del cliente, el número de ítems de la escala se reduce a la mitad y tiene mayor confiabilidad y validez que la del modelo IS SERVQUAL.

Luego (paso 3), teniendo como base la clasificación hecha por Kishore, Rao, Nam, K. Rajagopalan y Chaudhury (2003), se define una población de clientes actuales de servicios de TIC y se calcula el tamaño de la muestra mediante un muestreo aleatorio simple con distribución de probabilidad normal.

Posteriormente, para darle validez de contenido a la escala IS SERVPERF, se diseña una entrevista semiestructurada dirigida a expertos en servicios de TIC, basada en Galeano (2004). En total, se aplican siete entrevistas y se realiza un grupo focal con tres expertos (paso 4). Como resultado de este proceso, se hacen mejoras al instrumento en relación a la redacción de las preguntas y se añaden 15 ítems a la escala original.

Después, se diseñó una entrevista semiestructurada siguiendo a Galeano (2004) y se aplicó a nueve clientes de servicios de TIC (paso 5). Como resultado de esta actividad, se ajusta el instrumento obteniendo a una encuesta estructurada de 31 ítems. Finalmente, esta ultima se distribuye a 500 clientes de servicios de TIC (paso 6). 
Figura 1. Metodología para la construcción de la escala

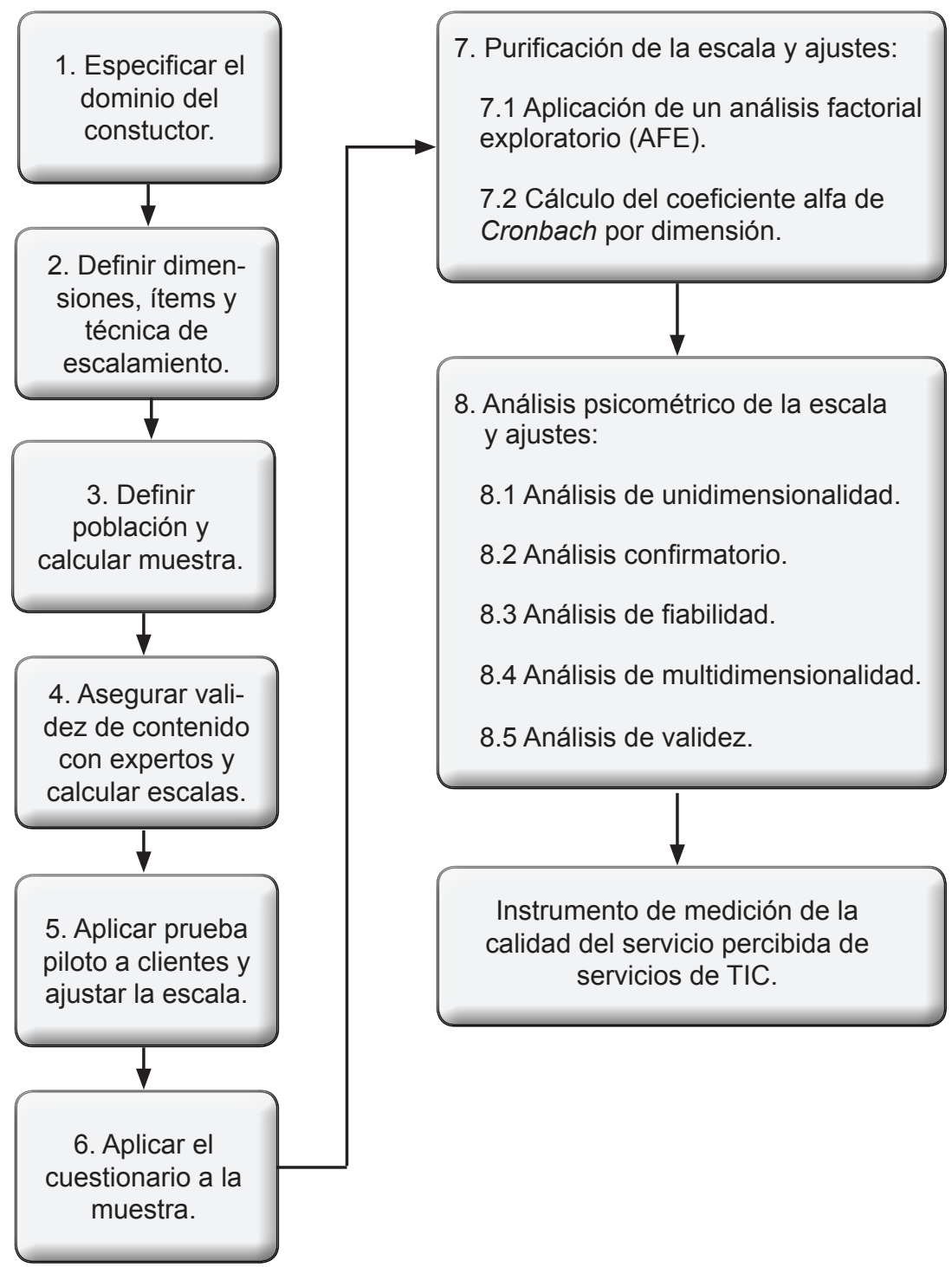

Fuente. Elaboración propia del autor.

En total, se recogen 153 encuestas válidas, de las cuales el $24 \%$ fueron respondidas por mujeres y el $76 \%$ por hombres. El $23 \%$ usan servicios de TIC en sus hogares y el $77 \%$ en su trabajo. Se presenta la distribución del número de personas encuestadas, de acuerdo al servicio de TIC que se evaluó, teniendo como base a Kishore, Rao, Nam, Rajagopalan y Chaudhury (2003) (Tabla 1). 
Tabla 1. Distribución de personas encuestadas por servicios de TIC evaluados

\begin{tabular}{|l|c|}
\hline \multicolumn{1}{|c|}{ Clientes de servicios de TIC } & $\begin{array}{c}\text { Porcentaje de } \\
\text { encuestados }\end{array}$ \\
\hline Comunicaciones & $41 \%$ \\
\hline Mantenimiento de Hardware & $33 \%$ \\
\hline Mantenimiento de Software & $11 \%$ \\
\hline Operaciones & $6 \%$ \\
\hline Administración de centros de datos & $5 \%$ \\
\hline Desarrollo de aplicaciones & $5 \%$ \\
\hline
\end{tabular}

Fuente. Elaboración propia del autor.

En el tabla 2, se presenta la ficha técnica de la aplicación de la escala.

Tabla 2. Ficha técnica de la aplicación de la encuesta

\begin{tabular}{|l|l|}
\hline \multirow{2}{*}{ Universo } & $\begin{array}{l}\text { Usuarios de servicios TIC que usen o hayan } \\
\text { usado en un periodo de menos de tres meses } \\
\text { en Cartagena, Colombia. }\end{array}$ \\
\hline Ámbito geográfico & Cartagena, Colombia \\
\hline Tamaño de la muestra & 153 \\
\hline Medio de aplicación de encuesta & Página Web \\
\hline Nivel de confianza & $95 \%$ \\
\hline Error muestral & $1.30 \%$ \\
\hline Periodo de recopilación de la & Del 10 al 17 de septiembre 2010 \\
\hline información & SPSS Versión $15.0 \mathrm{y}$ \\
\hline \multirow{2}{*}{ Programas estadísticos } & AMOS Versión 16.0 \\
\hline
\end{tabular}

Fuente. Elaboración propia del autor.

Con los resultados de la aplicación de la escala, se procede a hacer un análisis factorial exploratorio (AFE) y a calcular el coeficiente Alfa de Cronbach a cada una de las dimensiones obtenidas (paso 7) (tabla 3). 
Tabla 3. Resultados del AFE

\begin{tabular}{|c|c|c|c|c|c|}
\hline \multirow[t]{2}{*}{ Ítems } & \multicolumn{5}{|c|}{ Factores } \\
\hline & 1 & 2 & 3 & 4 & 5 \\
\hline p8 & 0.72 & & & & \\
\hline p9 & 0.65 & & & & \\
\hline p10 & 0.66 & & & & \\
\hline p11 & 0.77 & & & & \\
\hline p12 & 0.76 & & & & \\
\hline p15 & 0.67 & & & & \\
\hline p16 & 0.70 & & & & \\
\hline p17 & 0.63 & & & & \\
\hline p27 & 0.40 & & & & \\
\hline p29 & 0.56 & & & & \\
\hline p5 & & 0.53 & & & \\
\hline p18 & & 0.52 & & & \\
\hline p19 & & 0.52 & & & \\
\hline p21 & & 0.66 & & & \\
\hline p22 & & 0.70 & & & \\
\hline p23 & & 0.52 & & & \\
\hline p24 & & 0.67 & & & \\
\hline p25 & & 0.70 & & & \\
\hline p30 & & 0.53 & & & \\
\hline p31 & & 0.61 & & & \\
\hline $\mathrm{p} 1$ & & & 0.71 & & \\
\hline p2 & & & 0.78 & & \\
\hline $\mathrm{p} 13$ & & & 0.50 & & \\
\hline p14 & & & 0.42 & & \\
\hline p26 & & & 0.63 & & \\
\hline p28 & & & 0.62 & & \\
\hline p3 & & & & 0.69 & \\
\hline $\mathrm{p} 4$ & & & & 0.82 & \\
\hline p6 & & & & & 0.59 \\
\hline p7 & & & & & 0.61 \\
\hline p20 & & & & & 0.58 \\
\hline Fiabilidad & 0.91 & 0.89 & 0.85 & 0.61 & 0.73 \\
\hline Valor propio & 28.11 & 4.71 & 3.22 & 3.02 & 2.53 \\
\hline Varianza esperada (\%) & 42.09 & 7.05 & 4.82 & 4.53 & 3.80 \\
\hline
\end{tabular}

Fuente. Elaboración propia del autor. 
El AFE presentó un KMO de 0.911 y una prueba de esfericidad de Barlett con Chi-cuadrado de 2903.994; g.I. 465; p 0.000. Con base en los resultados del AFE, se obtienen cinco dimensiones que difieren del IS SERVQUAL, presentadas a continuación:

- La primera dimensión, se refiere a las percepciones que tiene el cliente de la disposición del proveedor a atender y ejecutar sus solicitudes de servicio de manera oportuna, organizada y confiable.

- La segunda dimensión, hace referencia a las percepciones del cliente sobre la experticia que demuestran los empleados del proveedor al atender sus requerimientos de servicio.

- La tercera dimensión, presenta la percepción del cliente de cumplimiento del proveedor con el servicio acordado, mediante el uso equipos actualizados.

- La cuarta dimensión, indica el atractivo visual que percibe el cliente de la sede del proveedor.

- La quinta dimensión, aborda la manera como el empleado se presenta a ejecutar la solicitud de servicio de un cliente y a la cortesía con que este percibe que lo trata. 


\section{Análisis psicométrico de la escala}
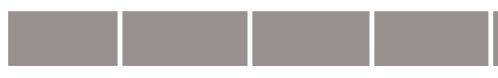

continuación, se presentan los análisis de unidimensionalidad, confirmatorio, de fiabilidad, multidimensionalidad y de validez de la escala.

\subsection{Análisis de unidimensionalidad}

Este análisis se aplica para identificar aquellos ítems que no pertenecen a su dimensión. Para esto, se realiza por cada una de las dimensiones resultantes, un análisis factorial de componentes principales con rotación varimax tal como sugieren Hair, Anderson, Tatham y Black (1998). (Tabla 4).

Tabla 4. Resultados de análisis de unidimensionalidad

\begin{tabular}{|c|c|c|c|c|}
\hline Dimensión & Ítem & $\begin{array}{c}\text { Carga } \\
\text { factorial }\end{array}$ & Valor propio & Varianza \\
\hline \multirow{10}{*}{ D1 } & preg8 & 0.771 & \multirow{10}{*}{5.713} & \multirow{10}{*}{$57.13 \%$} \\
\hline & preg9 & 0.807 & & \\
\hline & preg10 & 0.808 & & \\
\hline & preg11 & 0.853 & & \\
\hline & preg12 & 0.775 & & \\
\hline & preg15 & 0.664 & & \\
\hline & preg16 & 0.804 & & \\
\hline & preg17 & 0.799 & & \\
\hline & preg27 & 0.477 & & \\
\hline & preg29 & 0.73 & & \\
\hline
\end{tabular}


Tabla 4. Resultados de análisis de unidimensionalidad. (Continuación)

\begin{tabular}{|c|c|c|c|c|}
\hline \multirow{10}{*}{ D2 } & preg5 & 0.637 & \multirow{10}{*}{5.225} & \multirow{10}{*}{$52.25 \%$} \\
\hline & preg18 & 0.733 & & \\
\hline & preg19 & 0.773 & & \\
\hline & preg21 & 0.764 & & \\
\hline & preg22 & 0.78 & & \\
\hline & preg23 & 0.776 & & \\
\hline & preg24 & 0.711 & & \\
\hline & preg25 & 0.809 & & \\
\hline & preg30 & 0.662 & & \\
\hline & preg31 & 0.54 & & \\
\hline \multirow{6}{*}{ D3 } & preg1 & 0.749 & \multirow{6}{*}{3.438} & \multirow{6}{*}{$57.31 \%$} \\
\hline & preg2 & 0.82 & & \\
\hline & preg13 & 0.76 & & \\
\hline & preg14 & 0.687 & & \\
\hline & preg26 & 0.732 & & \\
\hline & preg28 & 0.788 & & \\
\hline \multirow{2}{*}{ D4 } & preg3 & 0.85 & \multirow{2}{*}{1.445} & \multirow{2}{*}{$72.25 \%$} \\
\hline & preg4 & 0.85 & & \\
\hline \multirow{3}{*}{ D5 } & preg6 & 0.802 & \multirow{3}{*}{1.946} & \multirow{3}{*}{$64.88 \%$} \\
\hline & preg7 & 0.818 & & \\
\hline & preg20 & 0.796 & & \\
\hline
\end{tabular}

Fuente. Elaboración propia del autor.

De acuerdo con los resultados de la tabla 4, las cinco dimensiones presentaron un nivel adecuado de unidimensionalidad, además, no se tiene que eliminar ningún ítem debido a que todas las cargas factoriales son superiores a 0.4 (Hair, Anderson, Tatham y Black, 1998).

\subsection{Análisis factorial confirmatorio}

De acuerdo a Uriel y Aldás (2005), esta técnica permite determinar la medida en que un modelo a Priori de relaciones entre variables observadas y latentes, se compara con la realidad de unos datos recogidos. 
De acuerdo a lo anterior, partimos del modelo resultante del análisis factorial exploratorio aplicado y luego, se eliminaran o introduc relaciones entre variables, hasta obtener un buen ajuste del modelo tal como sugiere Uriel y Aldás (2005).

Para desarrollar el modelo, seguiremos la estrategia propuesta por Hair, Anderson, Tatham y Black (1998), por que busca depurar una escala a partir de sucesivos análisis factoriales confirmatorios, para lo cual se eliminan de aquellos indicadores que no cumplan con los criterios de Jöreskog y Sörbom (1993), los cuales son:

- Criterio de convergencia débil, con el cual se eliminan aquellos indicadores que no presenten coeficientes de regresión factorial significativos (t student > 2,58; $p=0,01$ ).

- Criterio de convergencia fuerte, con este se suprimen los indicadores cuyos coeficientes estandarizados sean menores a 0,5.

- Finalmente, se descartan aquellos indicadores que menos aporten a la explicación del modelo teniendo como referente un $\mathrm{R} 2<0,3$.

se parte de un primer análisis factorial, confirmatorio y con los resultados obtenidos aplicamos el primer criterio; así, no se elimina ningún ítem debido a que todos cumplen con un t student $>2,58$ y $p=0,01$.

Luego, se aplica el segundo criterio y se eliminan los ítems preg3, preg5, preg15, preg27 y preg31. Es importante resaltar que como consecuencia de la eliminación de preg3, uno de los dos ítems que conforman la dimensión 4, se quita preg4, porque un solo indicador no puede caracterizar una dimensión, además, no podria ejecutarse el nuevo análisis factorial confirmatorio para verificar el tercer criterio de Jöreskog y Sörbom (1993). Realizado un tercer análisis factorial confirmatorio, se aplica el tercer criterio de Jöreskog y Sörbom (1993) y se excluyen los ítems preg1, preg6 y preg30; es así que se alcanza un ajuste satisfactorio del modelo.

Los resultados de los análisis factoriales confirmatorios aplicados, se presentan en la Tabla 5. 
Tabla 5. Resultados de los análisis factorial confirmatorio

\begin{tabular}{|l|l|l|l|l|}
\hline \multicolumn{1}{|c|}{ Índice } & \multicolumn{1}{|c|}{ Criterio } & $\begin{array}{c}\text { Resultado } \\
\text { AFC 1 }\end{array}$ & $\begin{array}{c}\text { Resultado } \\
\text { AFC 2 }\end{array}$ & $\begin{array}{c}\text { Resultado } \\
\text { AFC 3 }\end{array}$ \\
\hline X2 & & $\begin{array}{l}848,800(424) ; \\
p=0,000\end{array}$ & $\begin{array}{l}635,468(293) ; \\
p=0,000\end{array}$ & $\begin{array}{l}398,639(203) ; \\
p=0,000\end{array}$ \\
\hline X2/gl & $<3$ & 2,002 & 2,169 & 1,964 \\
\hline CFI & Próximo a 1 & 0,841 & 0,856 & 0,904 \\
\hline TLI & Próximo a 1 & 0,825 & 0,840 & 0,891 \\
\hline IFI & Próximo a 1 & 0,843 & 0,858 & 0,905 \\
\hline RMR & $\approx 0$ & 0,135 & 0,125 & 0,117 \\
\hline RMSEA & $<0.08$ & 0,081 & 0,088 & 0,080 \\
\hline
\end{tabular}

Fuente. Elaboración propia del autor.

\subsection{Análisis de fiabilidad}

Posteriormente, se realiza el cálculo del coeficiente alfa de Cronbach para comprobar la fiabilidad de la escala resultante (Tabla 6).

Tabla 6. Resultados de análisis de fiabilidad

\begin{tabular}{|c|c|}
\hline \multicolumn{2}{|c|}{ Fiabilidad } \\
\hline Dimensión & $\begin{array}{c}\text { Alfa de } \\
\text { Cronbach }\end{array}$ \\
\hline D1 & 0.92 \\
\hline D2 & 0.888 \\
\hline D3 & 0.828 \\
\hline D5 & 0.646 \\
\hline
\end{tabular}

Fuente. Elaboración propia de autor.

De acuerdo con lo anterior, sólo las dimensiones D1, D2 y D3 cumplen con el criterio mínimo del coeficiente alfa de Cronbach de 0.7 (Hair, Anderson, Tatham y Black, 1998; Zapata y Canet, 2008). La dimension 5 no cumple con el criterio de fiabilidad, por lo tanto se descarta. 


\subsection{Análisis de multidimensionalidad}

Se desarrolla la estrategia de modelos rivales de Hair, Anderson, Tathamy Black (1998); primero, se construye un modelo unidimensional (primer orden), que contiene en una sola dimensión todos los ítems de la escala. Luego, se aplica un análisis factorial confirmatorio y posteriormente, comparamos los índices de ajuste obtenidos, con los del modelo de cuatro dimensiones (segundo orden) que llevamos hasta este punto.

Para confirmar la multidimensionalidad del constructo, el modelo de cuatro dimensiones debe presentar mejor ajuste que el unidimensional (Steenkamp y Van Trijp, 1991).

La comparación de los índices de ajuste se presenta en la Tabla 7.

Tabla 7. Resultados de análisis de multidimensionalidad

\begin{tabular}{|l|l|l|l|}
\hline \multicolumn{1}{|c|}{ Índice } & \multicolumn{1}{|c|}{ Criterio } & $\begin{array}{l}\text { Unidimensional } \\
\text { (Primer orden) }\end{array}$ & $\begin{array}{l}\text { Multidimensional } \\
\text { (Segundo orden) }\end{array}$ \\
\hline $\mathrm{X}^{2}$ & & $\begin{array}{l}444.020(170) ; \\
\mathrm{p}=0.000\end{array}$ & $\begin{array}{l}348.079(167) ; \\
\mathrm{p}=0.000\end{array}$ \\
\hline $\mathrm{X} 2 / \mathrm{gl}$ & $<3$ & 2.612 & 2.084 \\
\hline $\mathrm{CFI}$ & Próximo a 1 & 0.857 & 0.905 \\
\hline $\mathrm{TLI}$ & Próximo a 1 & 0.840 & 0.892 \\
\hline $\mathrm{IFI}$ & Próximo a 1 & 0.858 & 0.906 \\
\hline $\mathrm{RMR}$ & $\approx 0$ & 0.134 & 0.119 \\
\hline RMSEA & $<0.08$ & 0.098 & 0.084 \\
\hline
\end{tabular}

Fuente. Elaboración propia del autor.

Debido a que el modelo de tres dimensiones (multidimensional) presenta mejores índices de ajuste, se afirma que el constructo es multidimensional.

\subsection{Análisis de validez}

Da acuerdo a Nunnally (1978), primero se realiza un análisis de validez de contenido y posteriormente uno del constructo. 


\subsubsection{Análisis de validez de contenido con expertos}

Para el análisis de validez contenido, se realizaron diez entrevistas semi estructuradas con expertos en servicios de TIC. Basándonos en Churchill (1979), Zapata y Canet (2008), Arribas (2004) y Hayes (1999), se solicita a los expertos evaluar la correspondencia entre los ítems de la escala y la calidad del servicio de TIC percibida, sobre lo cual afirmaron que es una buena aproximación y que sí podría medir el constructo.

\subsubsection{Análisis de validez de contenido con clientes}

Se realizaron diez entrevistas semiestructuradas con clientes de servicios de TIC, los cuales afirmaron que la escala mide la calidad de los servicios de TIC. Lo anterior, se realizó siguiendo las recomendaciones de Zapata y Canet (2008), Arribas (2004) y Hayes (1999).

\subsubsection{Análisis de validez del constructo}

Para realizar este análisis, se verificó si la escala tenia validez convergente y discriminante. De acuerdo a Bagozzi y Yi (1988), se tiene validez convergente cuando todos los coeficientes estandarizados son mayores que 0.5 y estadísticamente significativos al 0.01 . Los coeficientes estandarizados se presentan en la tabla 8 .

De acuerdo con los resultados presentados (Tabla 8), la escala presenta validez convergente. Para verificar la validez discriminante, se aplico la prueba de intervalos de confianza de Anderson y Gerbing (1988), para lo cual se estima la correlación entre las variables latentes del modelo y se verifica que en los intervalos de confianza no se encuentre incluido el valor 1 . A continuación, se presentan los resultados de la prueba de validez discriminante. 
Tabla 8. Coeficientes estandarizados

\begin{tabular}{|c|c|c|c|}
\hline \multicolumn{4}{|c|}{$\begin{array}{c}\text { Coeficientes estandarizados } \\
\text { (para todos p = 0.000) }\end{array}$} \\
\hline p29 & $<---$ & D1 & 0.706 \\
\hline p17 & $<---$ & D1 & 0.795 \\
\hline p16 & $<---$ & D1 & 0.768 \\
\hline p12 & $<---$ & D1 & 0.738 \\
\hline p11 & $<---$ & D1 & 0.827 \\
\hline p10 & $<---$ & D1 & 0.793 \\
\hline p9 & $<---$ & D1 & 0.805 \\
\hline p8 & $<---$ & D1 & 0.720 \\
\hline p25 & $<---$ & D2 & 0.788 \\
\hline p24 & $<---$ & D2 & 0.646 \\
\hline p23 & $<---$ & D2 & 0.795 \\
\hline p22 & $<---$ & D2 & 0.712 \\
\hline p21 & $<---$ & D2 & 0.706 \\
\hline p19 & $<---$ & D2 & 0.755 \\
\hline p18 & $<---$ & D2 & 0.727 \\
\hline p28 & $<---$ & D3 & 0.788 \\
\hline p26 & $<---$ & D3 & 0.680 \\
\hline p14 & $<---$ & D3 & 0.634 \\
\hline p13 & $<---$ & D3 & 0.761 \\
\hline p2 & $<---$ & D3 & 0.645 \\
\hline & & & \\
\hline
\end{tabular}

Fuente. Elaboración propia del autor .

Tabla 9. Resultados de la prueba de validez discriminante

\begin{tabular}{|c|c|}
\hline Pares de constructos & Intervalo de confianza \\
\hline D1-D2 & $0.795 ; 0.914$ \\
\hline D3-D1 & $0.744 ; 0.895$ \\
\hline D3-D2 & $0.764 ; 0.953$ \\
\hline
\end{tabular}

Fuente. Elaboración propia del autor.

La escala presenta validez discriminante de acuerdo a Anderson y Gerbing (1988) (Tabla 9). 


\section{Resultados}

Terminada la aplicación de la metodología propuesta, se obtiene un instrumento válido y confiable para medir la calidad del servicio percibida en servicios de TIC, el cual está compuesto por tres dimensiones y 20 ítems (Tabla 10).

Tabla 10. Instrumento de medida de la calidad del servicio de proveedores de TIC

\begin{tabular}{|l|l|}
\hline \multicolumn{1}{|c|}{ Dimensión } & \multicolumn{1}{c|}{ Ítems } \\
\hline \multirow{5}{*}{ Interés en el cliente } & $\begin{array}{l}\text { Su proveedor de servicios de TIC demuestra ser or- } \\
\text { ganizado en el desarrollo de las actividades relacio- } \\
\text { nadas con el servicio. }\end{array}$ \\
\cline { 2 - 3 } & $\begin{array}{l}\text { Los empleados de su proveedor de servicios de TIC } \\
\text { siempre están dispuestos a colaborarle. }\end{array}$ \\
\cline { 2 - 3 } & $\begin{array}{l}\text { Los empleados de su proveedor de servicios de TIC } \\
\text { atienden oportunamente las solicitudes de servicio de } \\
\text { sus usuarios. }\end{array}$ \\
\cline { 2 - 3 } & $\begin{array}{l}\text { Cuando los empleados de su proveedor de servicios } \\
\text { de TIC, a la hora de ejecutar el servicio, le confirman } \\
\text { que semorarán un determinado periodo de tiempo en } \\
\text { terminarlo, estos le cumplen. }\end{array}$ \\
\cline { 2 - 3 } & $\begin{array}{l}\text { Los empleados del proveedor de servicios de TIC } \\
\text { responsables de la ejecución de sus solicitudes de } \\
\text { servicio, le atienden en el tiempo que confirman hacerlo. }\end{array}$ \\
\cline { 2 - 3 } & $\begin{array}{l}\text { El proveedor de servicios de TIC le inspira confianza en } \\
\text { los servicios que le presta. }\end{array}$ \\
\cline { 2 - 3 } & $\begin{array}{l}\text { Cuando usted presenta un problema a su proveedor } \\
\text { de servicios de TIC, este muestra interés sincero en } \\
\text { resolverlo. }\end{array}$ \\
\cline { 2 - 3 } $\begin{array}{l}\text { Cuando el proveedor de servicios de TIC promete hacer } \\
\text { algo en un determinado tiempo, lo cumple. }\end{array}$ \\
\hline
\end{tabular}


Tabla 10. Instrumento de medida de la calidad del servicio de proveedores de TIC. (Continuación)

\begin{tabular}{|c|l|}
\hline \multirow{5}{*}{$\begin{array}{c}\text { Experticia de los } \\
\text { empleados }\end{array}$} & $\begin{array}{l}\text { Los empleados del proveedor de servicios de TIC entien- } \\
\text { den sus necesidades específicas. }\end{array}$ \\
\cline { 2 - 3 } & $\begin{array}{l}\text { El proveedor de servicios de TIC le brinda atención } \\
\text { personalizada. }\end{array}$ \\
\cline { 2 - 3 } & $\begin{array}{l}\text { Los empleados del proveedor de servicios de TIC } \\
\text { responsables por la ejecución de sus solicitudes de ser- } \\
\text { vicio, solucionan efectivamente los problemas que usted } \\
\text { reporta. }\end{array}$ \\
\cline { 2 - 3 } ele servicio.
\end{tabular}

Fuente. Elaboración propia del autor.

Tal y como lo muestra la Tabla 10, el instrumento resultante del proceso de validación consta de tres dimensiones que corresponden a:

- Interés en el cliente. Dimensión relacionada con el interés y disposición del proveedor de atender y ejecutar las solicitudes de servicio del cliente de manera oportuna, organizada y confiable. 
- Experticia de los empleados. Se refiere a los conocimientos y habilidades interpersonales que demuestran los empleados al atender los requerimientos de servicio de los clientes.

- Cumplimiento del proveedor. Dimensión que hace referencia al cumplimiento del proveedor, con el servicio acordado mediante el uso equipos actualizados.

Para profundizar en la comprensión de las tres dimensiones, se realizaron ocho entrevistas con clientes de servicios de TIC, quienes a partir de sus experiencias, permitieron comprender mejor cada una de ellas.

Sobre la dimensión interés en el cliente, los entrevistados manifestaron que el proveedor demuestra interés en el cliente cuando perciben que los empleados les atienden oportunamente, a través de los distintos canales de comunicación de los cuales disponen. Así mismo, cuando se percibe orden al recibir los requerimientos del cliente y al ejecutarlos, de acuerdo a un plan acordado entre las partes. Los entrevistados afirman que esto les demuestra calidad satisfactoria con el servicio que les ofrecen, lo que los lleva a recomendarlos y a tenerlos como única opción al tener la necesidad o el deseo de contratar un servicio de TIC.

Un cliente de servicios de TIC, manifestó que en ocasiones se ha sentido como un vendedor más de su proveedor, porque cada vez que se entera de una contratación en servicio de TIC, ya sea en la empresa donde trabaja o en otra, él hace un trabajo comercial espontáneo para que el contrato se lo den a su proveedor. Estas últimas afirmaciones de los clientes entrevistados son muy importantes porque indican fidelización de acción de acuerdo al modelo de fidelización de cuatro etapas de Oliver R. L., (1999).

De acuerdo a Oliver R. L., (1999), un consumidor que demuestre fidelidad de acción, sentirá un profundo compromiso para volver a comprar en el futuro el producto/servicio que le ofrezca su proveedor y además, realizará esa compra a pesar de los esfuerzos de mercadeo, para cambiar ese comportamiento, que realice la competencia.

Por otro lado, algunos entrevistados también afirmaron que el interés que demuestra un proveedor antes de firmar el contrato, se desvanece con el 
tiempo: "Al principio se les notaba el interés, pero después de un tiempo hay que rogarle al proveedor para que nos atienda y ejecuten el servicio requerido".

Dentro de las principales razones que manifestaron los entrevistados por las cuales el proveedor deja de demostrar interés en ellos están:

- El proveedor no tiene competencia en el mercado. En ese sentido el proveedor ofrece lo que le conviene.

- El empleado del proveedor no tiene conciencia de la importancia del cliente.

- El cliente adquiere un producto/servicio de TIC tan especializado, que se crea una fuerte dependencia hacia el proveedor. En este caso, el proveedor servirá como mejor le convenga.

- El cliente no representa grandes ingresos para el proveedor.

Los entrevistados manifestaron que para ellos las razones mencionadas les generaban insatisfacción, lo cual los ha llevado a terminar contratos, hablar mal del proveedor y no volver a tenerlos en cuenta para futuras contrataciones. De hecho, un entrevistado manifestó que éel no contrata servicios con proveedores de servicios TIC, si se da cuenta que algunos empleados de esos proveedores han trabajado en empresas donde el haya tenido malas experiencias.

Respecto a lo anterior, diversos autores concuerdan que la insatisfacción del cliente impide la fidelización a una empresa, la recompra del producto/ servicio y la recomendación del proveedor a otras personas (Lapré y Tsikriktsis, 2006; Oliver R.L., 1999). Lapré y Tsikriktsis (2006), resaltan la importancia de reconocer las insatisfacciones del cliente, ya que estas sirven como medio de aprendizaje para mejorar las percepciones que se tengan de la empresa.

Por otro lado, Homburg y Furst (2005), afirman que a pesar de las precauciones que se tomen en una empresa, se pueden presentar problemas entre esta y el cliente. Debido a esto, regularmente enfrentan quejas de 
los clientes y en ese sentido, el manejo que se le de es muy importante. De acuerdo a Homburg y Furst (2005), un mal manejo conlleva a que el cliente los evalue negativamente, mientras que un excelente manejo puede llegar a incrementar la satisfacción y fidelidad de un cliente, incluso, a un nivel más alto que el que tenía antes de la falla. De igual manera, resaltan que diversos estudios indican que invertir en la administración de las quejas de los clientes, puede llegar a dar un retorno sobre la inversión por encima del $100 \%$.

Sobre la dimensión experticia de los empleados, los entrevistados afirman que para ellos los principales representantes de toda una organización son las personas con las cuales tienen contacto, independientemente del departamento o área al que pertenezcan dentro de la empresa.

Los entrevistados afirman que ellos asumen que los empleados del proveedor tienen los suficientes conocimientos en TIC para ofrecer el servicio, pero manifiestan que muchas de las malas experiencias que han tenido, se deben al trato que los empleados les dan cuando hacen una solicitud de servicio.

Dentro de los malos tratos que han percibido mencionan los siguientes:

- Los empleados los hacen sentir como ignorantes.

- Les dicen mentiras o los confunden para justificarse aprovechándose, de su falta de conocimiento en TIC.

- No les tratan con respeto.

- Son groseros.

Lo anterior, ha traído consecuencias negativas para el proveedor, dentro de las cuales se identifican:

- No recomendar al proveedor a otras organizaciones.

- Hablar mal del proveedor.

- Hablar mal de los empleados del proveedor.

- No colaborarle al proveedor cuando este lo necesita.

Los entrevistados afirman que un buen trato de los empleados al cliente trae consecuencias positivas para un proveedor. De hecho, algunos 
manifestaron que han recomendado en repetidas ocasiones a un proveedor a causa del buen trato y de los conocimientos que han demostrado sus empleados.

Lo anterior resalta el rol fundamental de los empleados del proveedor en la formación de fidelidad de acción (Oliver R. L., 1999) en el cliente.

Guiltinan, Paul y Madden (1998), afirman que todos los departamentos y empleados de una empresa deben comprometerse y compartir la responsabilidad de la calidad para lograr satisfacer al cliente.

De acuerdo con lo anterior, los empleados no sólo deben demostrar amplios conocimientos sobre el servicio que ofrecen, sino tener habilidades para relacionarse adecuadamente con el cliente, de manera que incidan en la fidelidad del mismo.

Sobre la dimensión cumplimiento del proveedor, los entrevistados afirmaron que la finalidad de contratar un servicio de TIC es que le cumplan con los requerimientos que estos solicitan, los cuales son acordados con el proveedor.

De acuerdo a Grover, Joong Cheon, y T.C. Teng (1996), esto le permite al cliente enfocarse en su negocio principal y mejorar su competitividad en el mercado.

A pesar de la importancia mencionada para el cliente, algunos entrevistados manifestaron haber tenido complicaciones con el cumplimiento de los servicios contratados. Uno de ellos afirmó haber demandado a un proveedor por incumplimiento, lo cual exigió "la inversión obligada de recursos y tiempo que no estaban dentro de su presupuesto".

La persona afirmó, que no volvieron a contratar con estos proveedores y que en cada oportunidad que tiene "habla mal de ellos".

Es importante destacar que cada vez que los entrevistados mencionaron haber tenido malas experiencias con el servicio de TIC ofrecido por un proveedor, estas tuvieron un efecto opuesto en los indicadores de fidelidad del cliente identificados por Oliver (1999). 
De acuerdo a Oliver (1999), un cliente fiel habla bien de su proveedor y vuelve a comprar sus productos/servicios. Acá ocurre todo lo contrario, cuando se presentan insatisfacciones con el servicio y estas no se solucionan, los clientes entrevistados afirmaron hablar mal del proveedor y no volver a adquirir los servicios que este ofrece.

Finalmente, sobre el uso de equipos actualizados, algunos entrevistados manifestaron que para ellos no era necesario que el proveedor se los instalara, o los tuviera, siempre y cuando cumplieran con el servicio contratado, mientras que para otros, sí es necesario ya que manifestaron que pueden aprovechar los beneficios que los equipos actualizados traen.

Es de resaltar, que el uso de equipos actualizados les permite a los proveedores, no solo cumplir con el servicio acordado, sino ofrecerles otros a los clientes. 


\section{Conclusiones}

I presente trabajo busca construir una escala que mida la calidad del servicio de TIC percibida por los clientes de un proveedor externo, para que estos últimos diseñen y apliquen estrategias que les permitan mejorarla.

Para construir la escala, se hizo una revisión de la literatura del constructo calidad del servicio y de su aplicación en los sistemas de información, los cuales guardan estrecha relación con los servicios de TIC. La literatura presenta principalmente dos modelos, el IS SERVQUAL de Pitt, Watson, y Kavan (1995) y el IS SERVPERF de Van Dyke, Kappelman, y Prybutok (1997). Después de haber revisado los modelos y las críticas hechas a los mismos, se decide tomar el IS SERVPERF como modelo base para construir la escala.

Para el desarrollo de la escala, se aplicó una metodología de ocho pasos y como resultado se obtuvo un instrumento de tres dimensiones y 20 ítems. Las dimensiones son:

- Interés en el cliente.

- Experticia de los empleados.

- Cumplimiento del proveedor.

La aplicación de esta escala, permitiría a un proveedor de servicios de TIC diagnosticar la calidad del servicio que sus clientes le perciben y con base en los resultados, desarrollar estrategias para mejorarla; de esta manera, alcanzar los beneficios que manifestaron los entrevistados.

De igual manera, la escala permitiría evaluar a sus competidores y de esta manera diseñar e implementar estrategias que le permitan aumentar su competitividad en el mercado, aprovechando las falencias que encuentre en la calidad del servicio ofrecido en el mercado.

Así mismo, un emprendedor que quisiera ingresar en el sector de las TIC, puede agregarle valor a su emprendimiento incluyendo el constructo calidad 
del servicio percibido a su propuesta. De igual modo, el emprendedor también podría utilizar la escala para diagnosticar el mercado y con base en los resultados, mejorar su competitividad a partir de las deficiencias relacionadas con la calidad del servicio percibido, que encuentre en sus competidores.

Es importante resaltar, que la escala ofrece un diagnóstico de la calidad del servicio de un proveedor de TIC, pero se recomienda una vez obtenidos los resultados, se profundice en los hallazgos encontrados aplicando métodos de medición cualitativos tales como entrevistas y grupos focales. Esto, les permitirá comprender mejor los resultados obtenidos con la escala y mejorar la comprensión que se tenga del constructo en su organización y en el mercado.

Dentro de las limitaciones que presenta este trabajo, se encuentra que la escala representa mejor los servicios de TIC de comunicaciones y mantenimiento de Hardware. Esto debido a que el $74 \%$ de los encuestados evaluaron los dos servicios.

Para el resto de servicios, se sugiere contextualizarla siguiendo la metodología de ocho pasos utilizada. Esto permitirá tener una mejor aproximación del constructo para el resto de servicios. Para realizar la contextualización se puede tomar como base la escala propuesta.

Por otro lado, se recomienda aplicar un análisis de validez concurrente para relacionar la escala con otros constructos, tales como satisfacción del cliente y fidelización. Así, se permitirá afinar la escala y conocer la incidencia de las dimensiones en cada uno de esos constructos.

También se debe profundizar en cada una de las dimensiones propuestas en el modelo de calidad del servicio de TIC, con el fin de comprenderlas mejor y desarrollar indicadores que se aproximen mejor a su medición.

Finalmente, también se podrían realizar estudios longitudinales para comprobar la incidencia de la calidad del servicio de TIC en la capacidad competitiva de la organización y su crecimiento económico. 


\section{Referencias Bibliográficas}

Anderson, F. y Gerbing, D. W. (1988). Structural equation modeling in practice: a review and recommended two-step approach. Psychological Bulletin , 411-423.

Arbuckle, J. (1995). Amos v 16.0 User's guide. Amos v 16.0 User's guide. Chicago: Amos development corporation.

Arribas, M. (2004). Diseño y validación de cuestionarios. Matronas profesión. p 23-29.

Bacon, J. (1992). The use of decision criteria in selecting information systems/tecgnology investments. MIS Quarterly . p 335-353.

Beasley, M., Bradford, M. y Dehning, B. (2009). The value impact of strategic intent on firms engaged in information systems outsourcing. International Journal of Accounting Information Systems. p79-96.

Churchill, G. (1979). A paradigm for developing better measures of marketing constructs. Journal of marketing research. p 64-73.

Civera, M. (2008). Análisis de la relación entre calidad y satisfacción en el ámbito hospitalario en función del modelo de gestión establecido (Tesis de doctorado). Análisis de la relación entre calidad y satisfacción en el ámbito hospitalario en función del modelo de gestión establecido (Tesis de doctorado) . Castellón de la plana: Universitat Jaume.

Colmenares, O., y Saavedra, J. (2007). Aproximación teórica de los modelos conceptuales de la calidad del servicio. Técnica administrativa .

Cronin, J., y Taylor, S. (1992). Measuring service quality: A reexamination and extension. The Journal of marketing. p 55-68. 
Cronin, J. y Taylor, S. (1994). SERVPERF versus SERVQUAL: Reconciling performance-based and perceptions-minus-expectations measurement of service quality. The Journal of marketing. p 125-131.

Cronin, J. Brady, M., y Brand, R. (2002). Performance-only measurement of service quality: a replication and extension. Journal of Business Research. p 17-31.

DeLone, W. y McLean, E. (1992). Information systems success: The quest for the dependent variable. Information systems research. $p$ 60-95.

Deng, S. y Dart, J. (1994). Measuring market orientation: a multifactor, multi-item approach. Journal of Marketing Management. p, 725-742.

Duque, E. (2005). Revisión del concepto de calidad del servicio y sus modelos de medición. Innovar.p 64-80.

Duque, E., Cervera, A., y Rodríguez, R. (2006). Estudio bibliométrico de los modelos de medición del concepto de calidad percibida del servicio en Internet. Innovar. p 223-243.

Galeano, M. E. (2004). Diseño de proyectos en la investigación cualitativa. Medellín: Leticia Bernal.

Garcia Ruiz, M. E. (2003). Sistemas de información y nuevas tecnologías: influencias de las nuevas tecnologías en la estructura organizativa de la empresa cántabra (Tesis de doctorado). Cantabria: Universidad de Cantabria.

Gronroos, C. (1978). A service oriented approach to marketing of services. European Journal of marketing. p 588-601.

Grönroos, C. (1984). A service quality model and its marketing implications. European Journal of marketing. p 36-44.

Gronroos, C. (1982). An applied service marketing theory. European Journal of marketing. $p$ 30-41. 
Grover, V., Joong Cheon, M. y T.C. Teng, J. (1996). The Effect of Service Quality And Partnership on the Outsourcing of Information Systems Functions. Journal of Management Information Systems. p 89-116.

Guiltinan, J. P., Paul, G. W., Madden y J., T. (1998). Gerencia de marketing: Estrategías y programas. En J. P. Guiltinan, G. W. Paul, Madden, \& T. J., Gerencia de marketing: Estrategías y programas Bogotá: McGraw-Hill. p 11-12.

Hair, J. F., Anderson, R. E., Tatham, R. L. y Black, W. C. (1998). Multivariate data analysis 5a. ed. New Jersey: Prentice Hall.

Hayes, B. (1999). Como medir la satisfacción del cliente: Diseño de encuestas, uso y métodos de análisis estadístico. Mexico D.F.: Oxford.

Homburg, C., y Furst, A. (2005). How organizational complaint handling drives customer loyalty: An analysis of the mechanistic and the organic approach. Journal of marketing. p 95-114.

Jiang, J., Klein, G., y Carr, C. (2002). Measuring information system quality: SERVQUAL from the other side. MIS Quarterly. p 145-166.

Kahramana, C., Engin, O., Kabak, Ö. y Kaya, I. (2009). Information systems outsorucing decisions using a group decision-making approach. Enginnering applications of artificial intelligence. p 832841.

Kalb, L., Hoppen, N. y Henrique, J. L. (2009). Management of perception of information technology service quality. Journal of business research. p 876-882.

Kang, H. y Bradley, G. (2002). Measuring the performance of IT services: An assessment of SERVQUAL. International Journal of Accounting Information. p 151-164.

Kettinger, W. y Lee, C. (1994). Perceived Service Quality and User Satisfaction with the Information Services Function. Decision Sciences. p 737-766. 
Kettinger, W. y Lee, C. (1997). Pragmatic perspectives on the measurement of Information systems service quality. MIS quarterly. p 223-240.

Kettinger, W. y Lee, C. (2005). Zones of tolerance: Alternative Scales for Measuring Information Systems Service Quality. MIS Quarterly.p 607-623.

Kishore, R., Rao, H., Nam, K. Rajagopalan, S. y Chaudhury, A. (2003). A relationship perspective on IT outsourcing. Communications of the ACM. p 87-92.

Kohlmeyer, J. y Blanton, E. (2000). Improving IS service quality. Journal of information technology theory and application. p 1-10.

Kugyte, R. y Sliburyte, L. (2005). A standardized model of service provider selection criteria for different service types: a consumer-oriented approach. Engineering economics. p 56-63.

Lai, F., Griffin, M. y Babin, B. (2009). How quality, value, image, and satisfaction create loyalty at a Chinese telecom. Journal of Business Research. p 980-986.

Landrun, H., Zhang, X., Prybutok, V. y Peak, D. (2009). Measuring IS System service quality with SERVQUAL: User's perceptions of relative importance of the five SERVPERF dimensions. Informing Science: the international Journal of an emerging transdiscipline. $\mathrm{p}$ 18-35.

Lapré, M. A. y Tsikriktsis, N. (2006). Organizational learning curves for customer dissatisfaction: Heterogeneity across airlines. Management Science. p 352-366.

Laudon, K. y Laudon, J. (2008). Sistemas de información en los negocios globales actuales. En K. Laudon, \& J. Laudon, Sistemas de información gerencial: administración de la empresa digital. 10 edición.Mexico: Pearson educación. p. 4-32. 
Liou, J. y Chuang, Y. (2010). Developing a hybrid multi-criteria model for selection of outsourcing providers. Expert Systems with applications. p 3755-3761.

Lora, J. (2009). Estudio exploratorio criterios de selección de tecnologías de información en tres empresas cartageneras. Cartagena: Universidad Nacional de Colombia.

Lovelock, C. (1983). Classifying services to gain strategic marketing insights. Journal of marketing. p 9-20.

Mingers, J. (2006). Acritique of statisticalmodelling in management science from a critical realist perspective: Its role within multimethodology. The Journal of the operational research society. p 202-219.

Nunnally, J. (1978). Psychometric Theory. 2nd edition. New York: McGraw-Hill.

Oliver, R. (1980). A cognitive model of the antecedents and consequences of satisfaction decisions. Journal of marketing research. p 460-469.

Oliver, R. L. (1999). Whence costumer loyalty? The journal of marketing. p 33-44.

Oz, E. (2001). Alternativas para la adquisición de sistemas. En E. Oz, Administración de sistemas de información. 2a . edición.p 589-592. Mexico D.F.: Thomson Editores S.A. de C.V.

Paños, A. (1999). Las tecnologías de la información como fuente de ventajas competitivas: Una aproximación empírica (Tesis de doctorado). . Murcia: Universidad de Murcia.

Parasuraman, A., Zeithaml, V. y Berry, L. (1985). A conceptual model of service quality and its implications for future research. Journal of marketing. $p$ 41-50. 
Parasuraman, A., Zeithaml, V. y Berry, L. (1994). Reassessment of expectations as a comparison standard in measuring service quality: Implications for further research. The journal of marketing. p 111-124.

Parasuraman, A., Zeithaml, V. y Berry, L. (1988). SERVQUAL: A multipleitem scale for measuring consumer perceptions of service quality. Journal of retailing, 12-40.

Pitt, L., Watson, R. y Kavan, B. (1997). Measuring information systems service quality: Concerns for a complete canvas. MIS Quarterly , 209221.

Pitt, L., Watson, R. y Kavan, B. (1998). Measuring information systems service quality: Lessons from two longitudinal case studies. MIS Quarterly , 61-79.

Pitt, L., Watson, R. y Kavan, B. (1995). Service quality: A measure of information systems effectiveness. MIS Quarterly , 173-187.

Riascos, S. (2008). Modelo para la evaluación de la efectividad de la tecnología informática en el entorno empresarial. Revista de ingeniería e investigación. p 158-166.

Rodríguez, A. y Otálora, M. (2007). Calidad del servicio de salud: Una revisión a la literatura desde la perspectiva del marketing. Cuadernos de administración. p 237-258.

Ruiz, M., Pardo, A. y San Martín, R. (2010). Modelos de ecuaciones estructurales. Papeles del Psicólogo. p 34-45.

Shang, S. y Seddon, P. (2002). Assessing and managing the benefits of enterprise systems: the business managers's perspective. Information systems Journal. p 271-299.

SPSS. (2006). Manual del usuario de SPSS v 15.0. Chicago, EEUU: SPSS Inc.

Steenkamp, J. y Van Trijp, H. (1991). The use Lisrel in validating marketing constructs. International Journal of Research in Marketing. p 283-299. 
Teas, K. (1994). Expectations as a comparison standard in measuring service quality: An Assessment of a Reassessment. The journal of marketing. $\mathrm{p}$ 132-139.

Teas, K. (1993). Expectations, performance evaluation, and consumers' perceptions of quality. The Journal of marketing. $\mathrm{p}$ 18-34.

Uriel, E. yAldás, J. (2005). Análisis discriminante. En E. Uriel, \& A. Joaquín, Análisis multivariante aplicado. Madrid: Thomson. p 277-321

Uriel, E. y Aldás, J. (2005). Análisis factorial. En E. Uriel, \& A. Joaquín, Análisis multivariante aplicado. Madrid: Thomson. p 407-440

Uriel, E., y Aldás, J. (2005). Componentes principales. En E. Uriel, \& A. Joaquín, Análisis multivariante aplicado. Madrid: Thomson. p 365-394

Uriel, E. y Aldás, J. (2005). Ecuaciones estructurales: Análisis factorial confirmatorio. En E. Uriel, \& A. Joaquín, Análisis multivariante aplicado. Madrid: Thomson. p 442-488

Van Dyke, T., Kappelman, L. y Prybutok, V. (1997). Measuring Information systems Service Quality: Concerns on the Use of SERVQUAL Questionnaire. MIS Quartely. p 195-208.

Yang, Z., Cai, S., Zhou, Z. y Zhou, N. (2005). Development and validation of an instrument to measure user perceived service quality of information presenting Web portals. Information \& Management. $p$ 575-589.

Yost, J. y Harmon, K. (2002). Contracting for Information System Outsourcing with multiple Bidders. Journal of Information Systems. p 49-59.

Zapata, G. y Canet, M. T. (2008). Propuesta metodológica para la construcción de escalas de medición a partir de una aplicación empírica. Actualidades investigativas en educación. p 1-26.

Zeithaml, V. (1981). How consumer evaluation processes differ between goods and services. Marketing of services. p 186-189. 